\title{
Chloroquine Induces Human Mononuclear Phagocytes to Inhibit and Kill Cryptococcus neoformans by a Mechanism Independent of Iron Deprivation
}

\author{
Stuart M. Levitz, Thomas S. Harrison, Abdulmoneim Tabuni, and Xiuping Liu \\ The Evans Memorial Department of Clinical Research and the Department of Medicine, Boston Medical Center, Boston, Massachusetts 02118
}

\begin{abstract}
Infections due to Cryptococcus neoformans are common in AIDS patients. We investigated the effect of chloroquine, which raises the $\mathrm{pH}$ of phagolysosomes, on the anticryptococcal activity of mononuclear phagocytes. $C$. neoformans multiplied within monocyte-derived macrophages (MDM) in the absence of chloroquine but were killed with the addition of chloroquine. Ammonium chloride was also beneficial, suggesting that effects were mediated by alkalinizing the phagolysosome. Chloroquine inhibits growth of other intracellular pathogens by limiting iron availability. However, chloroquine-induced augmentation of MDM anticryptococcal activity was unaffected by iron nitriloacetate, demonstrating that chloroquine worked by a mechanism independent of iron deprivation. There was an inverse correlation between growth of $C$. neoformans in cell-free media and $\mathrm{pH}$, suggesting that some of the effect of chloroquine on the anticryptococcal activity of MDM could be explained by relatively poor growth at higher $\mathrm{pH}$. Chloroquine enhanced MDM anticryptococcal activity against all tested cryptococcal strains except for one large-capsule strain which was not phagocytosed. Positive effects of chloroquine were also seen in monocytes from both HIV-infected and -uninfected donors. Finally, chloroquine was therapeutic in experimental cryptococcosis in outbred and severe combined immunodeficient mice. Thus, chloroquine enhances the activity of mononuclear phagocytes against $C$. neoformans by ironindependent, $\mathrm{pH}$-dependent mechanisms and is therapeutic in murine models of cryptococcosis. Chloroquine might have clinical utility for the prophylaxis and treatment of human cryptococcosis. (J. Clin. Invest. 1997. 100:1640-1646.) Key words: chloroquine • macrophage • HIV • Cryptococcus $\bullet$ iron
\end{abstract}

\section{Introduction}

Infections due to the yeast Cryptococcus neoformans are a common cause of morbidity and mortality in persons infected with HIV (1-3). Even when successfully treated, AIDS patients with cryptococcosis are not cured and require lifetime

Address correspondence to Stuart M. Levitz, M.D., Room E336, Boston Medical Center, 88 E. Newton Street, Boston, MA 02118. Phone: 617-638-7904; FAX: 617-638-8070; E-mail: slevitz@med-med1.bu.edu

Received for publication 14 March 1997 and accepted in revised form 22 June 1997.

J. Clin. Invest.

(C) The American Society for Clinical Investigation, Inc. 0021-9738/97/09/1640/07 \$2.00

Volume 100, Number 6, September 1997, 1640-1646

http://www.jci.org therapy to prevent relapse. Thus, new therapeutic agents for treatment and prophylaxis of cryptococcal infections are needed. Ideal agents would have low toxicity, be relatively inexpensive, be easy to administer, and have a spectrum of activity that encompasses a range of pathogens commonly seen in individuals with HIV.

Chloroquine, a diprotic weak base which raises the endocytic and lysosomal $\mathrm{pH}$ of eukaryotic cells (4), is a widely used, relatively safe drug for the treatment of malaria, RA, and SLE. Its long half-life $(\sim 4 \mathrm{~d})$ in humans allows weekly administration. In human mononuclear phagocytes, chloroquine inhibits the growth of Legionella pneumophilia, Histoplasma capsulatum, and Francisella tularensis by limiting iron availability in the phagolysosome (4-6). This is accomplished because mobilization of iron from transferrin and ferritin, the two major sources of iron in the mononuclear phagocyte, is dependent upon an acidic environment. Iron dissociates from transferrin at acid $\mathrm{pH}$, becoming available to the microbe $(4$, 7). Thus, both ferric ions are released from transferrin at $\mathrm{pH} 5$ or below, and one ferric ion is released at $\mathrm{pH} 6$. At $\mathrm{pH} \geq 7$, ferric ions remain bound to transferrin. Iron availability from ferritin is thought to occur after $\mathrm{pH}$-dependent proteolysis in lysosomes (8-10). The ability of chloroquine to inhibit the growth of L. pneumophilia and $H$. capsulatum in human mononuclear phagocytes is reversed by ferric nitrilotriacetate (FeNTA ${ }^{1}$ an iron chelate that freely enters cells and releases iron independent of $\mathrm{pH}$ over the range of $\mathrm{pH}$ 5-8) but not holotransferrin (which releases iron only in an acidic environment) $(4,5,11,12)$. In addition to its beneficial effects in vitro, chloroquine proved therapeutic in a murine model of histoplasmosis (5).

Based on these studies, we hypothesized that chloroquine would similarly enhance the anticryptococcal capacity of human mononuclear phagocytes. We found that chloroquine did have a pronounced beneficial effect, although the mechanism was independent of iron deprivation and apparently mediated, at least in part, by the relatively poor growth of $C$. neoformans at higher $\mathrm{pH}$.

\section{Methods}

Materials. Unless otherwise noted, reagents were obtained from Sigma Chemical Co. (St. Louis, MO). All experiments were performed under conditions carefully designed to minimize endotoxin contamination, as described (13). RPMI 1640 and PBS were obtained from BioWhittaker, Inc. (Walkersville, MD) and contained $<0.005$ endotoxin $\mathrm{U} / \mathrm{ml}$. Pooled human serum (PHS) was obtained by combining serum from $\geq 10$ healthy donors under ice-cold conditions and

1. Abbreviations used in this paper: FeNTA, ferric nitriloacetate; MDM, monocyte-derived macrophages; NTA, nitriloacetate; PHS, pooled human serum; RITC, rhodamine isothiocyanate; SCID, severe combined immunodeficiency. 
storing it at $-70^{\circ} \mathrm{C}$ to preserve complement activity. PHS contained undetectable levels of endotoxin as measured by the Limulus lysate assay. Culture medium was RPMI 1640 supplemented with L-glutamine, penicillin, streptomycin, and 10\% PHS. NTA and FeNTA were prepared fresh for each experiment, as described, and filtersterilized before use $(4,11)$. Unless otherwise noted, cells were incubated at $37^{\circ} \mathrm{C}$ in humidified air supplemented with $5 \% \mathrm{CO}_{2}$

C. neoformans. Serotype A strains 6, 15, 98, 110, and 145 were gifts of Dr. Thomas Mitchell (Duke University, Durham, NC) (14). Strain ME was obtained from a patient at Boston Medical Center with cryptococcal meningitis (15). Except where otherwise noted, strain 145 was used for all studies presented herein. C. neoformans was grown on Sabouraud dextrose agar at $30^{\circ} \mathrm{C}$. Fungi were harvested after $4 \mathrm{~d}$ of growth, washed three times in PBS, counted using a hemocytometer, and resuspended at the desired concentration.

Isolation of PBMCs. Peripheral blood was obtained by venipuncture from healthy volunteers recruited from the staff, students, and faculty at Boston University Medical Center. The three HIV-seropositive donors were recruited from the immunodeficiency clinics at Boston Medical Center and had CD4 counts of 40, 80, and 150 cells $/ \mu 1$ blood. Blood donors did not have an acute concurrent illness or a history of cryptococcosis, and were not taking systemic antifungal medication or hematopoietic growth factors. Two of the three patients were taking antiretroviral treatment, although none was on protease inhibitors. The same donor was not used more than once.

Blood was anticoagulated with pyrogen-free heparin (Elkins-Sinn Inc., Cherry Hill, NJ) and centrifuged at $500 \mathrm{~g}$ for $15 \mathrm{~min}$. PBMCs were isolated by centrifugation of the leukocyte-rich buffy-coat cells on a Ficoll-Hypaque density gradient. PBMCs were washed twice in PBS and then enriched for monocytes by depletion of T cells by rosetting with neuraminidase-treated sheep red blood cells. Previous studies from our laboratory using this technique have demonstrated that $\sim 50 \%$ of the leukocytes from both HIV-seropositive and -seronegative donors stain positively for the monocyte markers CD14 and/ or CD13 by flow cytometric analysis. The remainder of the cells were mostly B and natural killer cells, with $<3 \% \mathrm{~T}$ cells (16). These cells are hereafter referred to as monocytes. Monocyte-derived macrophages (MDM) were obtained by culturing monocytes for $7 \mathrm{~d}$ in halfarea flat-bottomed wells containing $100 \mu \mathrm{l}$ culture medium. MDM were washed three times with RPMI 1640 before assay.

Assay of antifungal activity. Antifungal activity was determined as previously described $(17,18)$. Monocytes or MDM $\left(2 \times 10^{5}\right)$ and $C$. neoformans $\left(10^{4} \mathrm{CFU}\right)$ were incubated in wells of 96-well plates containing $100 \mu \mathrm{l}$ of culture medium supplemented with $10 \%$ (final concentration) PHS. Where indicated, chloroquine, NTA, FeNTA, holotransferrin, apotransferrin, and/or ammonium chloride, were added $30 \mathrm{~min}$ before the addition of C. neoformans. Plates were incubated for $18 \mathrm{~h}$, after which effector cells were lysed with $0.1 \%$ Triton X-100, and the number of CFU of C. neoformans was determined by dilution and spread plates on Sabouraud dextrose agar. This concentration of Triton X-100 had no effect on fungal viability. For each assay, sets of wells containing C. neoformans, medium, and PHS (but no effector cells) were incubated at $4^{\circ} \mathrm{C}$ to determine the inoculum of live organisms. We have found that replication of C. neoformans does not occur after incubation at $4^{\circ} \mathrm{C}$ for $18 \mathrm{~h}$. Triton $\mathrm{X}-100$ was added to the $4^{\circ} \mathrm{C}$ control wells as well as to the experimental wells before plating. Results are expressed as percent growth according to the formula, $\{(\mathrm{ex}-$ perimental CFU/inoculated CFU) -1$\} \times 100$. Thus, a value of zero indicates no net fungal growth. Values of 100,300 , and $700 \%$ would indicate an average of 1,2 , and 3 replications, respectively. A negative value indicates that the number of CFU decreased during the incubation period, and therefore that some fungal killing had occurred.

Binding assay. Binding of $C$. neoformans to MDM was determined using a modification of our previously described assay (19). MDM were incubated with C. neoformans in the presence or absence of chloroquine, using the exact same conditions as for the above antifungal activity except that the C. neoformans were labeled with rhodamine isothiocyanate (RITC) to facilitate their identification. After
$4 \mathrm{~h}$ of incubation, the percentage of fungi that were cell-associated (either surface-attached or internalized) was determined by epifluorescent microscopy. At least 200 yeast cells were counted per well.

Phagocytosis assay. Our previously described Uvitex assay was used to determine the percentage of cell-associated fungi that were internalized (phagocytosed) by the $\operatorname{MDM}(19,20)$. Briefly, MDM and RITC-labeled $C$. neoformans were coincubated for $4 \mathrm{~h}$ as described for the binding assay. Unbound organisms were washed free, and the cells were incubated with $0.1 \%$ Uvitex $2 \mathrm{~B}$ (also known as Fungiqual A and diaethanol; Specialty Chemicals for Medical Diagnostics, Kandern, Germany) for $1 \mathrm{~min}$. The wells were washed, fixed, and examined under an epifluorescent microscope. Internalized organisms stain with RITC alone, whereas extracellular organisms stain with both RITC and Uvitex. At least 100 cell-associated C. neoformans were scored per well.

Murine model of systemic cryptococcosis. 4-5-wk-old BALB/cByjSmn-Prkdc scid (severe combined immunodeficiency, SCID) female mice were purchased from The Jackson Laboratory (Bar Harbor, ME). 3-4-wk-old male outbred ICR mice were purchased from Harlan Sprague Dawley, Inc. (Indianapolis, IN). The mice were housed in microisolator cages and adapted to their environment before experimentation. Chloroquine was prepared in PBS, filter-sterilized, and injected intraperitoneally in a total volume of $200 \mu \mathrm{l}$. Control mice received $200 \mu \mathrm{l}$ PBS alone. A suspension of C. neoformans strain 145 was prepared in PBS as described above and injected intravenously in a total volume of $100 \mu$ l. Fungal inocula used were based on preliminary studies (data not shown) and previously described models of cryptococcosis (21).

Statistics. Except as noted below, means and SE were compared using the two-tailed paired $t$ test. Adjustments for significance based on multiple comparisons were made using Bonferroni's method. Statistical significance was considered to be achieved if the $P$ value multiplied by the number of comparisons was $<0.05$. Linear regression was used for experiments comparing fungal growth with $\mathrm{pH}$ of culture medium. The Mann-Whitney rank sum test was used to compare median survival in the mouse experiments. All statistical calculations were performed using SigmaStat Statistical Software (Jandel Scientific, San Rafael, CA).

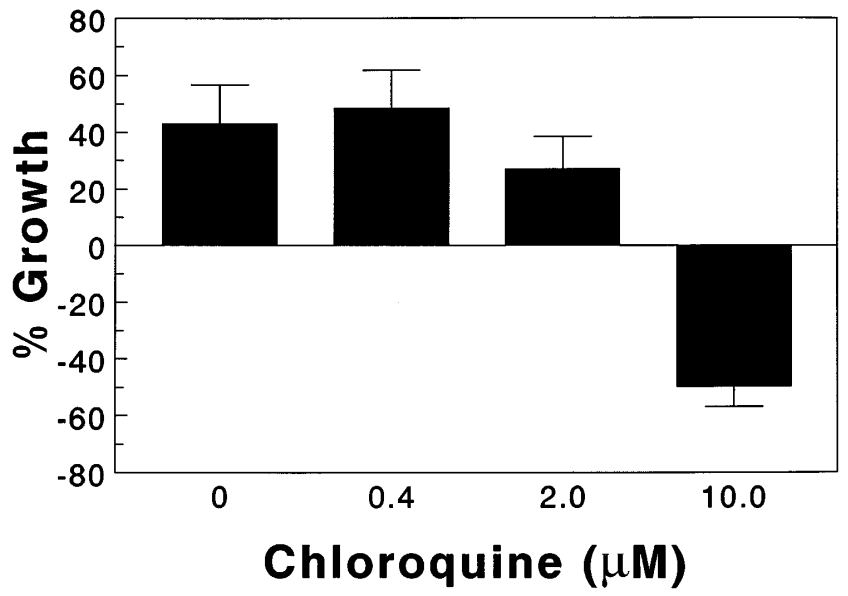

Figure 1. Dose-response effects of chloroquine on the activity of MDM against C. neoformans. MDM and C. neoformans were incubated for $18 \mathrm{~h}$ in the presence of the indicated concentration of chloroquine, after which antifungal activity was assayed as described in Methods. Data are means \pm SE of four separate experiments, each of which was performed in triplicate. $P<0.0001$ comparing no chloroquine with chloroquine at $10 \mu \mathrm{M} . P=0.059$ comparing no chloroquine with chloroquine at $2 \mu \mathrm{M}$. 


\section{Results}

Effect of chloroquine and ammonium chloride on activity of $M D M$ against $C$. neoformans. In initial experiments, the effect of varying concentrations of chloroquine on the anticryptococcal activity of MDM was assayed (Fig. 1). Consistent with published data from our laboratory and others $(22,23)$, in the absence of chloroquine, cryptococcal growth occurred in MDM. However, when $10 \mu \mathrm{M}$ chloroquine was present during the incubation, fungi were killed by MDM. Lower concentrations of chloroquine were not effective. If the incubation period of MDM and $C$. neoformans was shortened from 18 to $2 \mathrm{~h}$, the addition of $10 \mu \mathrm{M}$ chloroquine was without effect (data not shown). Consistent with data from others (24), chloroquine at concentrations up to $10 \mu \mathrm{M}$ had no direct effect on the growth of $C$. neoformans in culture medium without MDM (data not shown).

The antifungal activity of MDM was also enhanced in the presence of the weak base, ammonium chloride. In three independent experiments (each performed in triplicate), percent growth was $45.7 \pm 12.9$ and $3.6 \pm 6.0$ (means $\pm \mathrm{SE} ; P=0.002$ ) in the absence and presence, respectively, of $20 \mathrm{mM}$ ammonium chloride. Like chloroquine, ammonium chloride is a weak base which has been shown to raise the $\mathrm{pH}$ of lysosomal compartments $(4,25)$.

Contribution of iron deprivation to the effects of chloroquine. As discussed above, chloroquine inhibits the growth of L. pneumophilia and $H$. capsulatum by limiting iron availability in the phagolysosome, an effect that is reversed by FeNTA but not holotransferrin $(4,5)$. Accordingly, in the next set of experiments we examined whether FeNTA and holotransferrin could reverse the effects of chloroquine on the anticrypto-

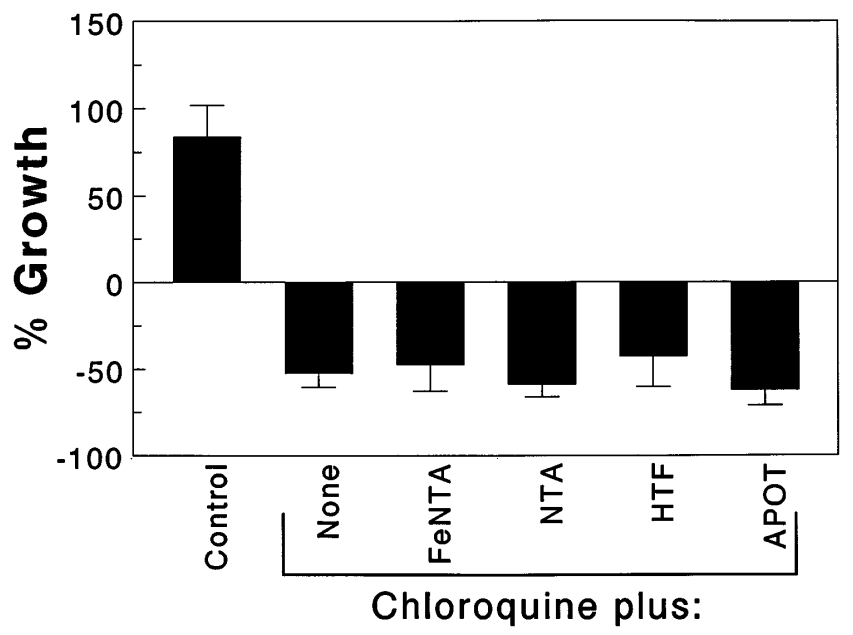

Figure 2. The effects of chloroquine are independent of iron deprivation. MDM were incubated for $18 \mathrm{~h}$ with $C$. neoformans strain 145 in the absence (Control) or presence of $10 \mu \mathrm{M}$ chloroquine. Groups that received chloroquine also received no additions (None), $50 \mu \mathrm{g} / \mathrm{ml}$ FeNTA, $50 \mu \mathrm{g} / \mathrm{ml} \mathrm{NTA}, 10 \mathrm{mg} / \mathrm{ml}$ holotransferrin $(H T F)$, or $10 \mathrm{mg} / \mathrm{ml}$ apotransferrin $(A P O T)$. Wells were incubated for $18 \mathrm{~h}$, after which antifungal activity was assayed as described in Methods. Data are means \pm SE of four separate experiments, each of which was performed in triplicate. There were no significant differences between any of the chloroquine-containing groups. $P<0.0001$ comparing no chloroquine with any group containing chloroquine.

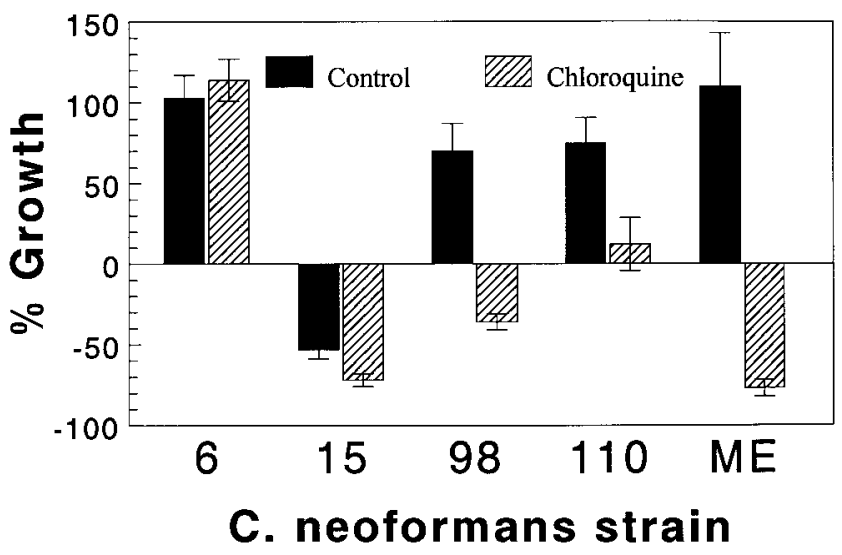

Figure 3. Strain-related variation in the effect of chloroquine on activity of MDM against C. neoformans. MDM were incubated for $18 \mathrm{~h}$ with the indicated strain of $C$. neoformans in the absence (Control) or presence of $10 \mu \mathrm{M}$ chloroquine, after which antifungal activity was assayed as described in Methods. Data are means \pm SE of two to three separate experiments, each of which was performed in triplicate. $P<$ 0.005 comparing no chloroquine with chloroquine for all of the strains except strain 6.

coccal activity of MDM. The iron-unsaturated forms of these compounds (NTA and apotransferrin) were included as controls. As in the experiments presented above, $10 \mu \mathrm{M}$ chloroquine significantly augmented the anticryptococcal activity of MDM (Fig. 2). FeNTA, NTA, holotransferrin, and apotransferrin had no significant effect on the anticryptococcal activity of chloroquine-treated MDM, strongly suggesting that chloroquine's effects are independent of iron deprivation. FeNTA, NTA, holotransferrin, and apotransferrin also had no significant effects on growth of $C$. neoformans in wells containing MDM but no chloroquine (data not shown).

Strain-related variation in the effect of chloroquine on activity of MDM against $C$. neoformans. The above experiments established the beneficial effects of chloroquine on the anticryptococcal activity of MDM against $C$. neoformans strain 145 . Because previous studies have demonstrated strain-related variations in certain responses of phagocytes to $C$. neoformans $(14,15,26,27)$, we next studied the activity of MDM against five additional strains of $C$. neoformans. Chloroquine enhanced the anticryptococcal activity of MDM against all of the strains tested except for strain 6 (Fig. 3). This strain is unique among the strains studied in that it is heavily encapsulated when grown on Sabouraud dextrose agar (14).

The effect of pH on cryptococcal growth. The above experiments established that chloroquine augments the antifungal activity of human MDM by a mechanism independent of iron deprivation. As it is known that chloroquine alkalinizes the phagolysosome, we hypothesized that the beneficial effects of chloroquine on MDM-mediated anticryptococcal activity are, at least in part, a direct result of the inability of $C$. neoformans to grow well at neutral and alkaline $\mathrm{pH}$ compared with acidic $\mathrm{pH}$. Accordingly, cryptococcal growth in cell-free tissue culture medium as a function of $\mathrm{pH}$ was determined for strains 145 and 6 (Fig. 4). The $\mathrm{pH}$ of the culture medium was adjusted with hydrochloric acid or sodium hydroxide, and remained within $0.2 \mathrm{pH} \mathrm{U}$ for the duration of the 18 -h incubation period. 


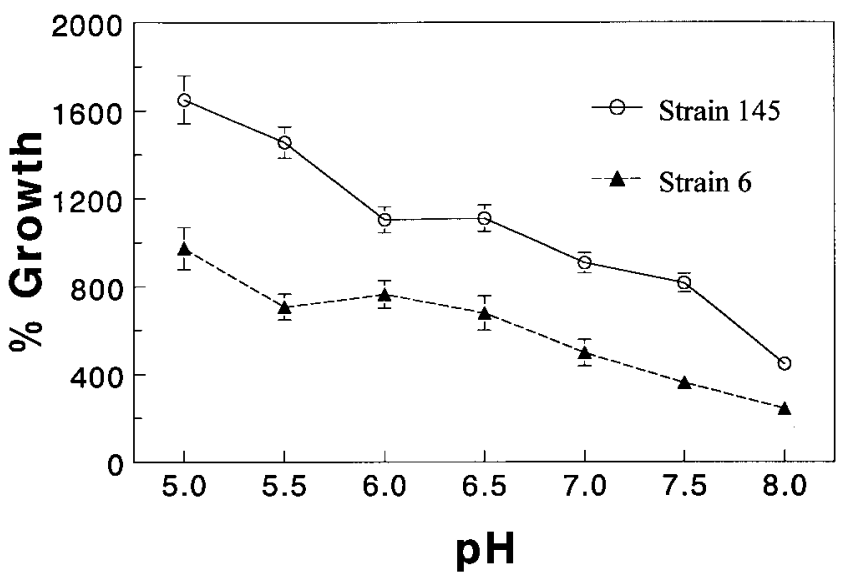

Figure 4. Effect of $\mathrm{pH}$ on growth of cryptococcal strains. Strains 145 and 6 were incubated in tissue culture medium adjusted to the indicated $\mathrm{pH}$ for $18 \mathrm{~h}$, and percent growth was measured, as described in Methods. There was a significant inverse correlation between $\mathrm{pH}$ and percent growth $(P<0.001$ for both strains by linear regression). Data are means \pm SE of two separate experiments, each of which was performed in triplicate.

For both strains tested, there was a significant inverse correlation between percent growth and $\mathrm{pH}$, with approximately fourfold greater growth seen at $\mathrm{pH} 5.0$ than at $\mathrm{pH} 8.0$.

Effect of chloroquine on binding and phagocytosis. As demonstrated in Figs. 3 and 4, despite an inverse correlation between growth and $\mathrm{pH}$, chloroquine failed to promote the antifungal activity of MDM against strain 6 . In contrast, chloroquine had a beneficial effect on all other tested strains. Strain 6 is a heavily encapsulated strain, and cryptococcal capsule is known to be antiphagocytic (28). Thus, we hypothesized that strain 6 , because of its heavy encapsulation, does not get phagocytosed effectively by MDM. Chloroquine increases the $\mathrm{pH}$ of intracellular compartments without having a significant effect on the $\mathrm{pH}$ of the extracellular culture medium. Thus,
pH-dependent effects of chloroquine on growth of $C$. neoformans would be expected to be limited to intracellular yeast cells. Accordingly, we next measured MDM-mediated binding and phagocytosis (internalization) of strains 145 and 6 in the presence and absence of chloroquine (Fig. 5). Chloroquine had no effect on binding and phagocytosis of either strain. For both strains, most of the $C$. neoformans added to the wells became cell-associated over the course of the 4-h assay, although there was a trend towards greater binding of strain 145 than strain 6. However, profound differences were seen when phagocytosis was examined: whereas $>80 \%$ of cell-associated strain 145 were phagocytosed by the MDM, $<20 \%$ of cellassociated strain 6 were actually internalized $(P<0.0001)$. The majority of yeast cells from strains 15, 98, 110, and ME were internalized by MDM (data not shown).

Effect of chloroquine on monocytes from HIV-seronegative and-seropositive donors. The next set of experiments was designed to answer two basic questions. First, does chloroquine work on monocytes in addition to MDM? Second, as most cases of cryptococcosis occur in the setting of HIV infection, does chloroquine have a beneficial effect on monocytes from HIV-infected donors? Accordingly, PBMCs were isolated from both HIV-seropositive and -seronegative donors and tested for anticryptococcal activity in the presence and absence of chloroquine (Fig. 6). Similar to its effects on MDM, chloroquine augmented the anticryptococcal capacity of freshly isolated monocytes. Beneficial effects were seen regardless of the HIV status of the blood donor.

Effect of chloroquine in murine models of cryptococcosis. The final set of experiments examined the effect of chloroquine on mice experimentally infected with $C$. neoformans. Two strains of mice were selected for study, immunocompetent (outbred) and immunodeficient (SCID), based on the types of patient populations that get cryptococcosis. Mice received $15 \mathrm{mg} / \mathrm{kg}$ of chloroquine every other day. This dosage was based on preliminary studies which determined that mice receiving doses $\geq 30 \mathrm{mg} / \mathrm{kg} / \mathrm{d}$ sometimes developed lethal toxicity from the chloroquine (data not shown). In both strains of mice, chloroquine was protective (Fig. 7).
A

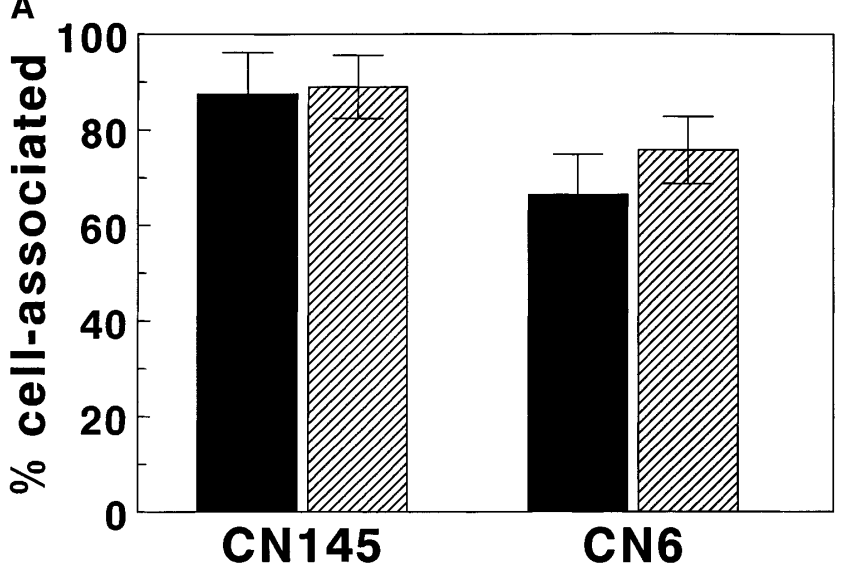

B

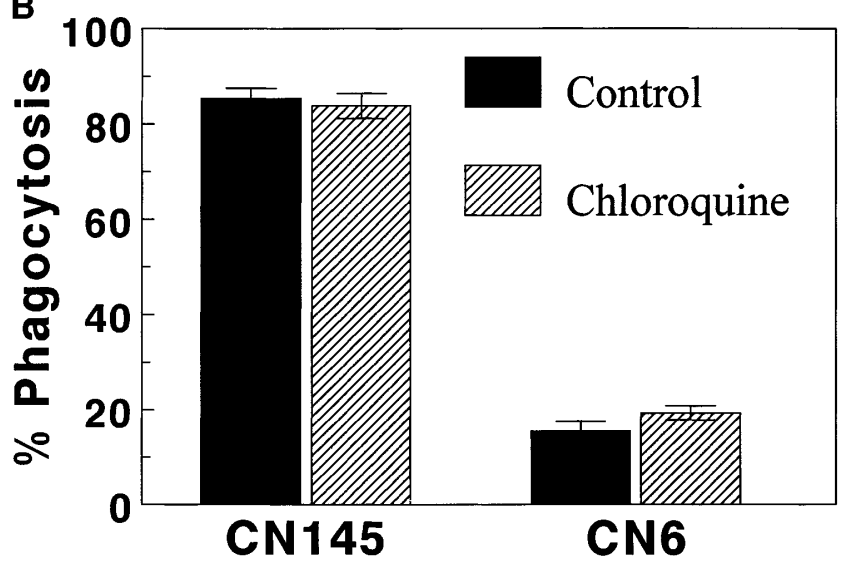

Figure 5. Binding and phagocytosis of strains 145 and 6. MDM were incubated for $4 \mathrm{~h}$ with either strain 145 or strain 6 of $C$. neoformans in the absence or presence of $10 \mu \mathrm{M}$ chloroquine, and then binding (cell-association) and phagocytosis (internalization) were determined as described in Methods. $(A)$ Percentage of $C$. neoformans which was cell-associated. (B) Percentage of cell-associated $C$. neoformans that was actually phagocytosed (internalized). $P<0.0001$ comparing phagocytosis of the two strains regardless of the presence of chloroquine. Results are means \pm SE of two separate experiments, each of which was performed in triplicate. 


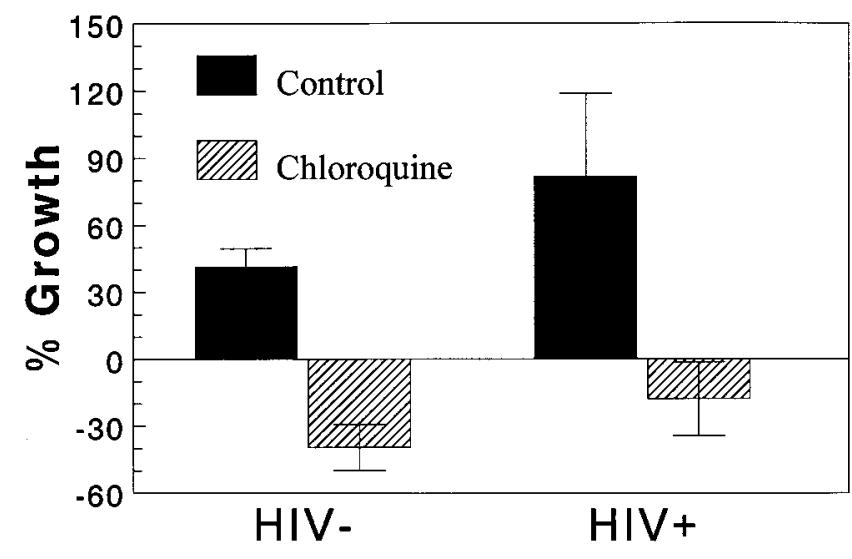

Figure 6. Effect of chloroquine on the anticryptococcal activity of monocytes from HIV-seronegative (HIV-) and seropositive $(\mathrm{HIV}+)$ donors. PBMCs from HIV - and HIV+ donors were incubated for $18 \mathrm{~h}$ with C. neoformans strain 145 in the absence (Control) or presence of chloroquine, after which antifungal activity was assayed as described in Methods. Data are means \pm SE of three separate experiments, each of which was performed in triplicate. $P<0.001$ comparing HIV - in the absence and presence of chloroquine. $P=$ 0.005 comparing $\mathrm{HIV}+$ in the absence and presence of chloroquine.

\section{Discussion}

The data presented demonstrate that chloroquine enhances the activity of freshly isolated monocytes and MDM against $C$. neoformans. Beneficial effects were seen in both HIV-seronegative and -seropositive donors. Moreover, chloroquine was therapeutic in two murine models of cryptococcosis. Taken together, these data suggest that chloroquine has potential as a novel therapeutic agent against cryptococcosis.

The inability of FeNTA to reverse the effects of chloroquine on the anticryptococcal activity of MDM argues strongly against a role for iron deprivation as a mechanism of action. $C$. neoformans is not known to produce siderophores, and its mechanism of iron acquisition is uncertain $(29,30)$. There is experimental evidence that $C$. neoformans can generate ferrous iron at the cell surface via reduction of ferric chelates, with the subsequent acquisition of the ferrous iron (30). However, chloroquine enhances the activity of MDM against $L$. pneumophilia (which also has an iron reductase system; reference 31) by $\mathrm{pH}$-dependent iron deprivation (4). Recently, a transferrin-like molecule with iron-binding properties has been purified from $C$. neoformans and postulated to play a role in iron uptake by this organism (32). Regardless of the mechanism of iron acquisition, chloroquine and its effects on $\mathrm{pH}$ do not appear to affect the ability of $C$. neoformans to obtain adequate iron in the MDM phagolysosome.

$\mathrm{pH}$ did have a significant, direct effect on growth of $C$. neoformans in cell-free culture medium. Howard, using $2 \%$ buffered proteose peptone medium, also found that the growth of C. neoformans is markedly inhibited in media with initial $\mathrm{pH}$ values slightly above neutrality (33). The ability of $C$. neoformans to grow better at acidic $\mathrm{pH}$ may give it a survival advantage in the phagolysosome and help explain the observation that under certain conditions $C$. neoformans grows as well if not better inside MDM than in tissue culture medium $(22,23)$.

Our data suggest that at least some of the effect of chloroquine on the activity of MDM against $C$. neoformans can be explained by the relatively poor growth of this fungus at higher $\mathrm{pH}$. However, even at $\mathrm{pH} 8.0, C$. neoformans still grew in cellfree culture medium, albeit slowly, whereas chloroquine treatment resulted in MDM developing fungicidal properties. Slowing down the growth of the organism could give time to enable the fungicidal mechanisms of the MDM to take effect. Such mechanisms as they apply to $C$. neoformans are unknown, although the MDM respiratory burst does not appear to be essential (our unpublished data). Human mononuclear phagocytes are known to possess several neutral proteinases (including elastase, cathepsin $\mathrm{G}$, at least two collagenases, and stromelysin), some of which are found in markedly increased amounts in MDM compared with monocytes $(34,35)$. This raises the possibility that in addition to slowing cryptococcal growth, raising the $\mathrm{pH}$ could result in more efficient antifungal

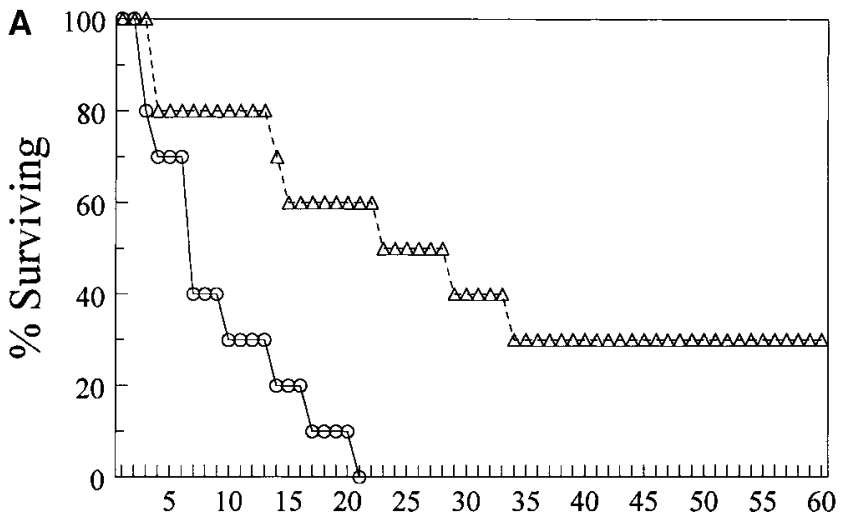

$\mathrm{D}$

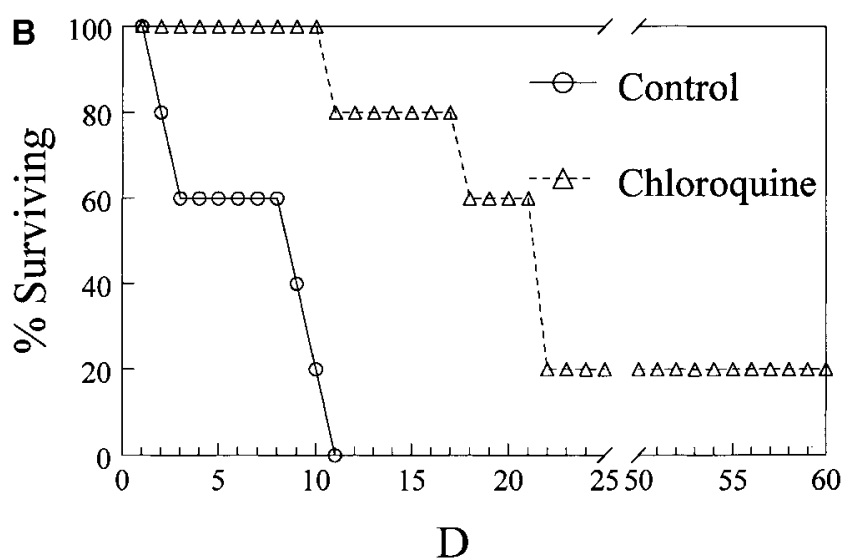

$\mathrm{D}$

Figure 7. Effect of chloroquine on survival of mice with experimental cryptococcosis. Outbred (10 per group, $A$ ) and SCID (5 per group, $B$ ) mice were treated with $15 \mathrm{mg} / \mathrm{kg}$ of chloroquine intraperitoneally every other day beginning $2 \mathrm{~d}$ before infection. Control mice received PBS alone. Mice were injected intravenously with $3 \times 10^{6}$ (outbred) or $10^{5}$ (SCID) C. neoformans strain 145 , and survival was determined daily. $P=$ 0.02 and 0.008 comparing median survival of chloroquine and control groups by Mann-Whitney rank sum test for outbred and SCID mice, respectively. 
activity by optimizing the $\mathrm{pH}$ of certain antimicrobial MDM enzymes. If so, this would be in contradistinction to the situation with other intracellular microbes studied, where acidification is thought to enhance antimicrobial activity of phagocytes, in part because many hydrolytic enzymes are active only at low $\mathrm{pH}(36)$.

Chloroquine has also been shown to inhibit secretion of certain immunoactive substances, including $\mathrm{TNF}-\alpha$ and $\mathrm{PGE}_{2}$ $(37,38)$. Other biological effects of chloroquine have been described that are purportedly $\mathrm{pH}$-independent, including inhibition of calcium release from macrophage intracellular stores and intercalation into DNA (39). However, ammonium chloride also enhanced the anticryptococcal activity of MDM. As both ammonium chloride and chloroquine raise the $\mathrm{pH}$ of phagolysosomes $(4,25)$, these findings strongly suggest that the effects of chloroquine on MDM activity against $C$. neoformans are mediated by an increase in the $\mathrm{pH}$ of the phagolysosome. The potency of ammonium chloride was less than that of chloroquine, a finding that could be due to the capacity of ammonium chloride (but not chloroquine) to inhibit phagolysosome fusion (40).

Chloroquine had beneficial effects on all C. neoformans strains tested except for strain 6 , which is a heavily encapsulated strain that was poorly phagocytosed by MDM. This finding is consistent with the hypothesis that, because chloroquine works on intracellular compartments, internalization of $C$. neoformans is necessary for chloroquine's activity. The medical correlate of this finding is that in clinical cases of cryptococcosis, where both intracellular and extracellular fungi are seen (41), chloroquine would only be expected to have effects on the intracellular pool of fungi. Theoretically, then, administration of anticryptococcal antibody (which would be expected to increase phagocytosis of $C$. neoformans) should work synergistically in combination with chloroquine. Clinical trials of a humanized anticryptococcal mAb have been proposed (42).

Chloroquine was protective in two distinct murine models of cryptococcosis, one that used immunocompetent outbred mice and the other that used SCID mice. These two mouse strains were chosen because they serve as models for most human hosts with cryptococcosis $(1-3,21)$. Thus, the outbred mice mimic the clinical situation of cryptococcosis in the immunocompetent patient, whereas SCID mice mimic the clinical situation in AIDS and other disorders of cell-mediated immunity. Recently, intracerebral administration of chloroquine was shown to increase median survival time and decrease yeast growth in the brains of mice given a lethal intracerebral inoculum of $C$. neoformans (43). Newman et al. (5) also found that chloroquine was therapeutic in a murine model of histoplasmosis, although they used a substantially higher dosage of chloroquine than was used in our studies.

Chloroquine has excellent bioavailability after oral administration, and, at usual therapeutic dosages, serum concentrations of $1-1.5 \mu \mathrm{M}$ are obtained $(5,44)$. However, chloroquine accumulates in phagocytes, and it has been estimated that cellular levels comparable to those found during antirheumatic therapy are achieved by the in vitro incubation of cells with $100 \mu \mathrm{M}$ chloroquine (45). This concentration is 10 -fold greater than the concentration of chloroquine necessary to empower mononuclear phagocytes for enhanced anticryptococcal activity in our experiments. Moreover, chloroquine is greatly concentrated in certain tissues, where $\mathrm{mM}$ concentrations can be obtained. Taken together with our findings that chloroquine was protective in animal models, our data lend support for clinical trials evaluating chloroquine for adjunctive treatment and prophylaxis of cryptococcosis. In addition to its onceweekly oral dosage and low toxicity, chloroquine has a potential advantage over antifungal agents (such as amphotericin B and fluconazole) in that it does not have a direct effect on the fungus. Thus, the likelihood of resistance developing should be relatively small. Moreover, chloroquine may be beneficial for other intracellular pathogens that affect individuals with impaired cell-mediated immunity, as experimental data with several of these pathogens suggest $(4-6,46)$. However, because chloroquine can interfere with effective antigen-processing (47), the greatest benefit might be in those patients with severely impaired $\mathrm{T}$ cell function, particularly individuals with AIDS and CD4+ T cell counts $<100 / \mu l$ blood. Antigen-processing is probably superfluous in such patients due to their inability to mount a $\mathrm{T}$ cell response. Interestingly, the chloroquine analogue hydrochloroquine has been shown to inhibit replication of HIV-1 in infected T cells and monocytes (48). In addition, in a small clinical trial, HIV-infected individuals treated with hydrochloroquine had a significant decrease in their total plasma levels of HIV-1 RNA (49).

\section{Acknowledgments}

The authors thank Dr. Alan Sugar, Dr. Simon Newman, and Dr. George Deepe for helpful discussions.

This work was supported by grants RO1-AI37532 and RO1AI25780 from the National Institutes of Health. T.S. Harrison is the recipient of the Infectious Diseases Society of America Pfizer-Roerig Divisional Fellowship in Mycology.

\section{References}

1. Diamond, R.D. 1995. Cryptococcus neoformans. In Mandell, Douglas and Bennett's Principles and Practice of Infectious Diseases. G.L. Mandell, J.E. Bennett, and R. Dolin, editors. Churchill-Livingstone Inc., New York. 23312340.

2. Powderly, W.G. 1993. Cryptococcal meningitis and AIDS. Clin. Infect. Dis. 17:837-842.

3. Levitz, S.M. 1991. The ecology of Cryptococcus neoformans and the epidemiology of cryptococcosis. Rev. Infect. Dis. 13:1163-1169.

4. Byrd, T.F., and M.A. Horwitz. 1991. Chloroquine inhibits the intracellular multiplication of Legionella pneumophila by limiting the availability of iron. A potential new mechanism for the therapeutic effect of chloroquine against intracellular pathogens. J. Clin. Invest. 88:351-357.

5. Newman, S.L., L. Gootee, G. Brunner, and G.S. Deepe, Jr. 1994. Chloroquine induces human macrophage killing of Histoplasma capsulatum by limiting the availability of intracellular iron and is therapeutic in a murine model of histoplasmosis. J. Clin. Invest. 93:1422-1429.

6. Fortier, A.H., D.A. Leiby, R.B. Narayanan, E. Asafoadjei, R.M. Crawford, C.A. Nancy, and M.S. Mitzer. 1995. Growth of Francisella tularensis LVS in macrophages: the acidic intracellular compartment provides essential iron required for growth. Infect. Immun. 63:1478-1483.

7. Dautry-Varsat, A., A. Ciechanover, and H.F. Lodish. 1983. $\mathrm{pH}$ and the recycling of transferrin during receptor-mediated endocytosis. Proc. Natl. Acad. Sci. USA. 80:2258-2262.

8. Starke, P.E., J.D. Gilbertson, and J.L. Farber. 1985. Lysosomal origin of the ferric iron required for cell killing by hydrogen peroxide. Biochem. Biophys. Res. Commun. 133:371-379.

9. Sibille, J.C., H. Kondo, and P. Aisen. 1989. Uptake of ferritin and iron bound to ferritin by rat hepatocytes: modulation by apotransferrin, iron chelators and chloroquine. Biochim. Biophys. Acta. 1010:204-209.

10. Sibille, J.C., M. Ciriolo, H. Kondo, R.R. Crichton, and P. Aisen. 1989. Subcellular localization of ferritin and iron taken up by rat hepatocytes. Biochem. J. 262:685-688.

11. Bates, G.W., and J. Wernicke. 1971. The kinetics and mechanism of iron(3) exchange between chelates and transferrin. IV. The reaction of transferrin with iron(3) nitrilotriacetate. J. Biol. Chem. 246:3679-3685.

12. Olakanmi, O., J.B. Stokes, and B.E. Britigan. 1994. Acquisition of iron bound to low molecular weight chelates by human monocyte-derived macrophages. J. Immunol. 153:2691-2703. 
13. Levitz, S.M., A. Tabuni, H. Kornfeld, C.C. Reardon, and D.T. Golenbock. 1994. Production of tumor necrosis factor-alpha in human leukocytes stimulated by Cryptococcus neoformans. Infect. Immun. 62:1975-1981.

14. Miller, M.F., and T.G. Mitchell. 1991. Killing of Cryptococcus neoformans strains by human neutrophils and monocytes. Infect. Immun. 59:24-28.

15. Levitz, S.M., E.A. North, Y. Jiang, S. Nong, H. Kornfeld, and T.S. Harrison. 1997. Variables affecting production of monocyte chemotactic factor 1 from human leukocytes stimulated with Cryptococcus neoformans. Infect. Immun. 65:903-908.

16. Harrison, T.S., and S.M. Levitz. 1996. Role of IL-12 in PBMC responses to fungi in persons with and without HIV infection. J. Immunol. 156:4492-4497.

17. Harrison, T.S., H. Kornfeld, and S.M. Levitz. 1995. The effect of infection with human immunodeficiency virus on the anticryptococcal activity of lymphocytes and monocytes. J. Infect. Dis. 172:665-671.

18. Levitz, S.M. 1991. Activation of human peripheral blood mononuclear cells by interleukin-2 and granulocyte-macrophage colony-stimulating factor to inhibit Cryptococcus neoformans. Infect. Immun. 59:3393-3397.

19. Levitz, S.M., and A. Tabuni. 1991. Binding of Cryptococcus neoformans by human cultured macrophages. Requirements for multiple complement receptors and actin. J. Clin. Invest. 87:528-535.

20. Levitz, S.M., A. Tabuni, T.R. Kozel, R.S. MacGill, R.R. Ingalls, and D.T. Golenbock. 1997. Binding of Cryptococcus neoformans to heterologously expressed human complement receptors. Infect. Immun. 65:931-935.

21. Clemons, K.V., R. Azzi, and D.A. Stevens. 1996. Experimental systemic cryptococcosis in SCID mice. J. Med. Vet. Mycol. 34:331-335.

22. Levitz, S.M., and T.P. Farrell. 1990. Growth inhibition of Cryptococcus neoformans by cultured monocytes: role of the capsule, opsonins, the culture surface, and cytokines. Infect. Immun. 58:1201-1209.

23. Diamond, R.D., and J.E. Bennett. 1973. Growth of Cryptococcus neoformans within human macrophages in vitro. Infect. Immun. 7:231-236.

24. Wang, Y., and A. Casadevall. 1994. Susceptibility of melanized and nonmelanized Cryptococcus neoformans to nitrogen- and oxygen-derived oxidants. Infect. Immun. 62:3004-3007.

25. Krogstad, D.J., and P.H. Schlesinger. 1987. Acid-vesicle function, intracellular pathogens, and the action of chloroquine against Plasmodium falciparum. N. Engl. J. Med. 317:542-549.

26. Kozel, T.R., G.S. Pfrommer, A.S. Guerlain, B.A. Highison, and G.J. Highison. 1988. Strain variation in phagocytosis of Cryptococcus neoformans: dissociation of susceptibility to phagocytosis from activation and binding of opsonic fragments of C3. Infect. Immun. 56:2794-2800.

27. Small, J.M., T.G. Mitchell, and R.W. Wheat. 1986. Strain variation in composition and molecular size of the capsular polysaccharide of Cryptococcus neoformans serotype A. Infect. Immun. 54:735-741.

28. Levitz, S.M. 1994. Macrophage Cryptococcus interactions. In Macrophage Pathogen Interactions. B.S. Zwilling and T.K. Eisenstein, editors. Marcell Dekker, Inc., New York. 533-543.

29. Jacobson, E.S., and M.J. Petro. 1987. Extracellular iron chelation in Cryptococcus neoformans. J. Med. Vet. Mycol. 25:415-418.

30. Vartivarian, S.E., R.E. Cowart, E.J. Anaissie, T. Tashiro, and H.A. Sprigg. 1995. Iron acquisition by Cryptococcus neoformans. J. Med. Vet. Mycol. 33:151-156.

31. Johnson, W., L. Varner, and M. Poch. 1991. Acquisition of iron by Legionella Pneumophila: role of iron reductase. Infect. Immun. 59:2376-2381.

32. Tesfa-Selase, F., and R.J. Hay. 1996. Partial characterization and identification of a transferrin-like molecule of pathogenic yeast Cryptococcus neofor- mans. J. Gen. Appl. Microbiol. 42:61-70.

33. Howard, D.H. 1961. Some factors which affect the initiation of growth for Cryptococcus neoformans. J. Bacteriol. 82:430-435.

34. Nathan, C.F. 1987. Secretory products of macrophages. J. Clin. Invest. 79:319-326.

35. Campbell, E.J., J.D. Cury, S.D. Shapiro, G.I. Goldberg, and H.G. Welgus. 1991. Neutral proteinases of human mononuclear phagocytes. Cellular differentiation markedly alters cell phenotype for serine proteinases, metalloproteinases, and tissue inhibitor of metalloproteinases. J. Immunol. 146:1286-1293.

36. Eissenberg, L.G., W.E. Goldman, and P.H. Schlesinger. 1993. Histoplasma capsulatum modulates the acidification of phagolysosomes. J. Exp. Med. 177:1605-1611.

37. Zhu, X., W. Ertel, A. Ayala, M.H. Morrison, M.M. Perrin, and I.H. Chaudry. 1993. Chloroquine inhibits macrophage tumour necrosis factor-alpha mRNA transcription. Immunology. 80:122-126.

38. Ertel, W., M.H. Morrison, A. Ayala, and I.H. Chaudry. 1992. Chloroquine attenuates hemorrhagic shock-induced immunosuppression and decreases susceptibility to sepsis. Arch. Surg. 127:70-75.

39. Misra, U.K., G. Gawdi, and S.V. Pizzo. 1997. Chloroquine, quinine and quinidine inhibit calcium release from macrophage intracellular stores by blocking inositol 1,4,5-trisphosphate binding to its receptor. J. Cell. Biochem. 64:225-232.

40. Hart, P.D., and M.R. Young. 1991. Ammonium chloride, an inhibitor of phagosome-lysosome fusion in macrophages, concurrently induces phagosomeendosome fusion, and opens a novel pathway: studies of a pathogenic mycobacterium and a nonpathogenic yeast. J. Exp. Med. 174:881-889.

41. Baker, R.D., and R.K. Haugen. 1955. Tissue changes and tissue diagnosis in cryptococcosis. A study of 26 cases. Am. J. Clin. Path. 25:14-24.

42. Zebedee, S.L., R.K. Koduri, J. Mukherjee, S. Mukherjee, S. Lee, D.F. Sauer, M.D. Scharff, and A. Casadevall. 1994. Mouse-human immunoglobulin G1 chimeric antibodies with activities against Cryptococcus neoformans. Antimicrob. Agents Chemother. 38:1507-1514.

43. Mazzolla, R., R. Barluzzi, A. Brozzetti, J.R. Boelaert, T. Luna, S. Saleppico, F. Bistoni, and E. Blasi. 1997. Enhanced resistance to Cryptococcus neoformans infection induced by chloroquine in a murine model of meningoencephalitis. Antimicrob. Agents Chemother. 41:802-807.

44. Mackenzie, A.H. 1983. Pharmacologic actions of 4-aminoquinoline compounds. Am. J. Med. 75:5-10.

45. French, J.K., N.P. Hurst, M.L. O’Donnell, and W.H. Betts. 1987. Uptake of chloroquine and hydroxychloroquine by human blood leucocytes in vitro: relation to cellular concentrations during antirheumatic therapy. Ann. Rheum. Dis. 46:42-45.

46. Crowle, A.J., and M.H. May. 1990. Inhibition of tubercle bacilli in cultured human macrophages by chloroquine used alone and in combination with streptomycin, isoniazid, pyrazinamide, and two metabolites of vitamin D3. Antimicrob. Agents Chemother. 34:2217-2222.

47. Collins, H.L., and G.J. Bancroft. 1991. Encapsulation of Cryptococcus neoformans impairs antigen-specific T-cell responses. Infect. Immun. 59:38833888 .

48. Sperber, K., T.H. Kalb, V.J. Stecher, R. Banerjee, and L. Mayer. 1993. Inhibition of human immunodeficiency virus type 1 replication by hydroxychloroquine in T cells and monocytes. AIDS Res. Hum. Retroviruses. 9:91-98.

49. Sperber, K., M. Louie, T. Kraus, J. Proner, E. Sapira, S. Lin, V. Stecher, and L. Mayer. 1995. Hydroxychloroquine treatment of patients with human immunodeficiency virus type 1. Clin. Ther. 17:622-636. 